\title{
Reporte de caso
}

\section{Impacto de la variación en la altura sobre el nivel del mar, en los parámetros clínicos y hemodinámicos en hipertensión arterial pulmonar: a propósito de un caso}

Enrique Saul Sanabria Pérez ${ }^{1, a^{*}}$, José Guillermo Ercilla Sánchez ${ }^{2, b}$, Oscar Aguirre Zurita ${ }^{1, a}$

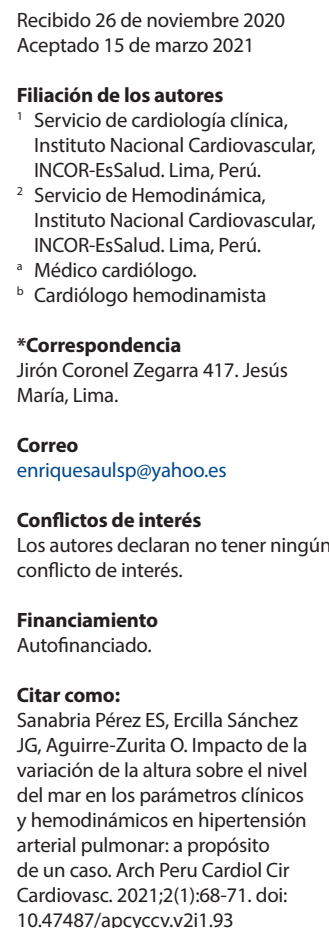

\section{RESUMEN}

El seguimiento clínico de la hipertensión arterial pulmonar en nuestro país, en el que los pacientes proceden de distintas altitudes sobre el nivel del mar, nos obliga a descartar hipertensión pulmonar en relación con la exposición crónica a grandes alturas, descrita en el tercer grupo de la clasificación internacional. Al revisar las variaciones hemodinámicas de la presión pulmonar con ejercicio en altura con respecto al nivel del mar en pacientes sanos, esta es mayor en altura, lo que explica la mayor sintomatología en altura. Presentamos un caso clínico de hipertensión pulmonar que mostró valores diferentes en la hemodinámica de altura y a nivel del mar.

Palabras clave: Hipertensión Pulmonar; Hipoxia; Insuficiencia Cardiaca (fuente: DeCS BIREME).

\section{ABSTRACT}

Impact of variation in altitude above sea level on clinical and hemodynamic parameters in pulmonary arterial hypertension: case report

Clinical monitoring of pulmonary arterial hypertension in our country, in which patients come from different altitudes above sea level, forces us to rule out pulmonary hypertension in relation to chronic exposure to high heights, described in third group of international classification. When reviewing the hemodynamic variations in pulmonary pressure with exercise at altitude with respect to sea level in healthy patients, this is greater in height, this would explain that the patient with pulmonary arterial hypertension is more symptomatic while living at a higher altitude above sea level.

Key words: Hypertension, Pulmonary; Hypoxia; Heart Failure (source: MeSH NLM). 
$\mathrm{L}$ a hipertensión arterial pulmonar (HAP) es una enfermedad rara, progresiva y de alta mortalidad. Las recomendaciones actuales en la estrategia de tratamiento agresivo y los tratamientos combinados tempranos se asocian en especial con una mejora de los resultados ${ }^{(1)}$. Actualmente, más de 140 millones de personas en el mundo viven sobre los 2500 metros sobre el nivel del mar (m s.n.m.) ${ }^{(2)}$ y en los andes del Perú habita el 38\% de la población nacional. Existe una relación directa entre altitud sobre el nivel del mar y el valor de la presión media de la arteria pulmonar (PmAP), considerándose que por encima de los 3000 a 3500 m de altitud existe hipertensión pulmonar (HP) asintomática en el poblador nativo de la altura ${ }^{(2,3)}$.

\section{Presentación del caso}

Paciente mujer de 41 años, natural y procedente de Huancayo, ciudad ubicada a $3200 \mathrm{~m}$ de altitud; es referida a nuestro centro ubicado en la ciudad de Lima a nivel del mar, en marzo de 2012, con el diagnóstico de HAP. El tiempo de enfermedad era aproximadamente de un año, caracterizada por disnea de clase funcional NYHA (New York Heart Association) IIIII y dos episodios de síncope. Fue evaluada por un neumólogo en el hospital de origen quien descartó enfermedad pulmonar asociada; a su ingreso recibía medicación: furosemida $40 \mathrm{mg} /$ día, espironolactona $25 \mathrm{mg} /$ día y digoxina $0,125 \mathrm{mg}$ /día.

Al examen físico se encontraron signos de insuficiencia cardiaca derecha (ingurgitación yugular, hepatomegalia, ascitis y edema de miembros inferiores), saturación de oxígeno $95 \%$ al aire ambiente. Se realizó gammagrafía de perfusión pulmonar sin defectos de perfusión, exámenes para enfermedades autoinmunes negativos, perfil tiroideo normal, prueba de ELISA para infección por el virus de inmunodeficiencia humana (VIH) negativo, ecografía abdominal sin hallazgos de hipertensión portal.

En la ecocardiografía se encontró función sistólica del ventrículo izquierdo preservada, no hipertrofia ventricular izquierda, aurícula izquierda no dilatada, cavidades derechas dilatadas e insuficiencia tricuspídea severa, ausencia de comunicaciones anormales entre cavidades o grandes vasos. Se realizó prueba de caminata de seis minutos, dosaje de proBNP y cateterismo cardiaco con menos de 7 días a nivel del mar (Tabla 1), la prueba de vasorreactividad con adenosina demostró disminución de la PAP de $96 / 38$ media de $56 \mathrm{mmHg}$ a 63/26 con una media de $43 \mathrm{mmHg}$. Con estos hallazgos, la paciente es diagnosticada con hipertensión arterial pulmonar del grupo 1 idiopática, con lo que se prescribe sildenafilo 50 mg tres veces al día.
La paciente es reevaluada en enero del 2013, se encontraba en clase funcional II, no volvió a presentar síncope ni signos de insuficiencia cardiaca derecha, la prueba de caminata de 6 min fue de 324 metros con escala de Borg de disnea 2. En el ecocardiograma, las cámaras derechas estaban dilatadas con función del ventrículo derecho preservada e insuficiencia tricuspidea severa. Al no cumplir el objetivo terapéutico, la conducta habitual es agregar a la terapia específica antagonistas de endotelina (terapia combinada). En vista de que durante la anamnesis manifestaba que en el lugar de origen presentaba disnea clase funcional III, en la ciudad de Lima esta mejoraba a clase funcional II y ante las dificultades administrativas para el inicio de terapia combinada, se recomendó cambiar lugar de residencia a menos de $1500 \mathrm{~m}$ de altitud. Al siguiente control, viviendo por debajo de esa altitud, presentó mejoría de clase funcional en julio de 2015 (clase funcional l) y prueba de caminata de 6 min en 443 metros, escala de Borg de disnea 1. Además, en la ecocardiografía se evidenció reducción importante de los diámetros y área de ventrículo derecho y aurícula derecha, respectivamente, insuficiencia tricupidea ligera y pro-BNP 162 $\mathrm{pg} / \mathrm{mL}$. En marzo de 2017, viviendo más de un año a nivel del mar, se le realizó cateterismo cardiaco derecho (Tabla 1), permanecía en clase funcional I y en terapia solo con sildenafilo $50 \mathrm{mg}$, tres veces día.

En abril 2019 migra a España manteniéndose en clase funcional I. Fue evaluada en hospital Universitario Donostie San Sebastián en donde el tratamiento actual es amlodipino 5 mg día. El 10 de enero 2020 se le realizó cateterismo cardiaco con PAP basal de $120 / 43 \mathrm{mmmHg}$, media de $69 \mathrm{mmHg}$. El test de vasorreactividad fue positivo; la PAP llegó a $63 / 25 \mathrm{mmHg}$. media $38 \mathrm{mmHg}$; sin hipotensión sistémica ni cada del gasto cardiaco. La fracción de eyección del ventrículo derecho por resonancia magnética actual es de $46.3 \%$.

\section{Discusión}

Muchas personas viven a gran altitud y realizan sus actividades normalmente; sobre los 2500 m viven más de 140 millones de personas; de estos, 80 millones en Asia y 35 millones en los Andes, con una mayor densidad sobre los $3500 \mathrm{~m}^{(2)}$. Al revisar las guías actuales en la clasificación de hipertensión pulmonar, se hace referencia en el grupo 3, a la exposición crónica a grandes altitudes ${ }^{(1)}$, en nuestro continente la conocemos como el mal de montaña crónico ${ }^{(2)}$, esta condición está asociada con policitemia e hipoxemia, que no estaban presentes en la paciente. La hipertensión pulmonar disminuye con el descenso al nivel del mar, luego de 2 años se puede ver normalización de las presiones pulmonares, lo cual es una norma en pacientes con mal de montaña crónico ${ }^{(4,5)}$. 
Tabla 1. Características clínicas y hemodinamicas de la paciente según evolución en el tiempo y lugar de residencia

\begin{tabular}{|c|c|c|c|c|}
\hline Lugar de residencia & $\begin{array}{c}\text { Huancayo } 3200 \mathrm{~m} \text { de } \\
\text { altitud } \\
\text { Junio } 2012\end{array}$ & $\begin{array}{c}\text { Huancayo } 3200 \text { m de } \\
\text { altitud } \\
\text { Enero } 2012\end{array}$ & $\begin{array}{c}\text { Lima } 100 \text { m de altitud } \\
\text { Julio } 2015\end{array}$ & $\begin{array}{c}\text { Lima } 100 \text { m de altitud } \\
\text { Marzo } 2017\end{array}$ \\
\hline Clase funcional NYHA & III & II & I & I \\
\hline Síncope & Sí & No & No & No \\
\hline $\begin{array}{l}\text { Insuficiencia cardiaca } \\
\text { derecha }\end{array}$ & Sí & No & No & No \\
\hline $\begin{array}{l}\text { Prueba de caminata- } \\
\text { Borg }\end{array}$ & $310 m-3$ & $324 m-2$ & $443 \mathrm{~m}-1$ & $429 m-0$ \\
\hline Ecocardiograma & $\begin{array}{c}\text { AD } 32 \mathrm{~cm}^{2} \text { VD } 34 \mathrm{~mm} \\
\text { IT severa Tapse } 16 \mathrm{~mm} \\
\text { Grad VD/AD } 65 \mathrm{mmHg} \\
\text { No derrame } \\
\text { pericárdico }\end{array}$ & $\begin{array}{c}\text { AD } 27 \mathrm{~cm}^{2} \text { VD } 44 \mathrm{~mm} \\
\text { IT severa Tapse } 20 \mathrm{~mm} \\
\text { Grad VD/AD } 76 \mathrm{mmHg} \\
\text { No derrame } \\
\text { pericárdico }\end{array}$ & $\begin{array}{c}\text { AD } 29 \mathrm{~cm}^{2} \text { VD } 32 \mathrm{~mm} \\
\text { IT severa Tapse18 mm } \\
\text { Grad VD/AD } 42 \\
\text { mmHg } \\
\text { No derrame } \\
\text { pericárdico }\end{array}$ & $\begin{array}{l}\text { AD } 26 \mathrm{~cm}^{2} \text { VD } 42 \mathrm{~mm} \\
\text { IT severa Tapse } 20 \mathrm{~mm} \\
\text { Grad VD/AD } 43 \mathrm{mmHg} \\
\text { No derrame } \\
\text { pericárdico }\end{array}$ \\
\hline $\begin{array}{l}\text { Cateterismo cardiaco; } \\
\text { PAD-PAPm mmHg }\end{array}$ & $10-56$ & & & $8-40$ \\
\hline IC - RVP (uW) & $1,91-15,2$ & & & $2.2-7.24$ \\
\hline pro BNP pg/mL & 1002 & 920 & 162 & 488 \\
\hline Tratamiento & Inicia sildenafilo & Sildenafilo & Sildenafilo & Sildenafilo \\
\hline
\end{tabular}

AD: aurícula derecha. VD: ventrículo derecho. PAD: presión en aurícula derecha. IC: índice cardiaco. RVP: resistencia vascular pulmonar. RVS: resistencia vascular pulmonar. IT: insuficiencia tricupidea. uW: unidades Woods.

No se han reportado casos clínicos en el que se documente mejoría clínica, ecocardiográfica y hemodinámica de pacientes con hipertensión arterial pulmonar idiopática, con la sola variación de lugar de residencia de gran altitud a un lugar de baja altitud. Hay una relación directa representada por una curva parabólica entre el nivel de altura sobre el nivel del mar y el grado de la PAPm. De tal forma que por encima de los 3000 a 3500 m existe HP en el poblador nativo de la altura ${ }^{(6)}$.

En los estudios realizados con cateterismo cardiaco a nivel del mar, los estudios a baja carga duplican el gasto cardiaco e incrementan la PAPm en un 50\%. En la altura, el gasto cardiaco se duplica como a nivel del mar, pero la PAPm se incrementa en $100 \%$ o más a pesar del discreto ejercicio ${ }^{(5,7)}$.
La paciente no tenía criterios diagnósticos de mal de montaña crónico, por lo que consideramos que pertenece al grupo I de la clasificación. Planteamos el diagnóstico de hipertensión arterial pulmonar idiopática en una paciente que nació y vivió en la altura.

\section{Conclusiones}

Si tenemos en cuenta los hallazgos del caso clínico, podríamos recomendar que los pacientes que viven en zonas de altura que tienen el diagnóstico de hipertensión pulmonar deben migrar a lugares de baja altitud como lugar de residencia habitual.

\section{Referencias bibliográficas}

1. Galiè N, Humbert M, Vachiery J-L, Gibbs S, Lang I,Torbicki A, et al. 2015 ESC/ERS Guidelines for the diagnosis and treatment of pulmonary hypertension: The Joint Task Force for the Diagnosis and Treatment of Pulmonary Hypertension of the European Society of Cardiology (ESC) and the European Respiratory Society (ERS) Endorsed by: Association for European Paediatric and Congenital Cardiology (AEPC), International Society for Heart and Lung Transplantation (ISHLT). Eur Respir J. 2015;46(4):903-75. doi: 10.1183/13993003.01032-2015.
2. León-Velarde F, Maggiorini M, Reeves JT, Aldashev A, Asmus I, Bernardi $\mathrm{L}$, et al. Consensus statement on chronic and subacute high altitude diseases. High Alt Med Biol. 2005;6(2):147-57. doi: 10.1089/ ham.2005.6.147.

3. Neupane M, Swenson ER. High-Altitude Pulmonary Vascular Diseases. Adv Pulm Hypertens. 2017;15(3):149-57. doi: 10.21693/1933-088X15.3.149. 
4. Penaloza $D$, Arias-Stella J. The heart and pulmonary circulation at high altitudes: healthy highlanders and chronic mountain sickness. Circulation. 2007;115(9):1132-46. doi: 10.1161/ CIRCULATIONAHA.106.624544.

5. Sime F, Peñaloza D, Ruiz L. Bradycardia, increased cardiac output, and reversal of pulmonary hypertension in altitude natives living at sea level. Br Heart J. 1971;33(5):647-57. doi: 10.1136/hrt.33.5.647.
6. Banchero N, Sime F, Peñaloza D, Cruz J, Gamboa R, Marticorena E. Pulmonary Pressure, Cardiac Output, and Arterial Oxygen Saturation during Exercise at High Altitude and at Sea Level. Circulation. 1966;33(2):249-62. doi: 10.1161/01.CIR.33.2.249

7. Mirrakhimov AE, Strohl KP. High-altitude Pulmonary Hypertension: an Update on Disease Pathogenesis and Management. Open Cardiovasc Med J. 2016;10:19-27. doi: 10.2174/1874192401610010019. 